\title{
Ações multiprofissionais de cuidados paliativos em unidades de terapia intensiva:
}

\section{uma revisão integrativa}

\author{
Multiprofessional palliative care actions in intensive care units: an integrative review \\ Actuaciones multiprofesionales de cuidados paliativos en las unidades de cuidados intensivos: una \\ revisión integradora
}

Recebido: 05/11/2021 | Revisado: 12/11/2021 | Aceito: 18/11/2021 | Publicado: 28/11/2021

\author{
Marilia Alves Furtado \\ ORCID: https://orcid.org/0000-0003-2672-9679 \\ Universidade Estadual do Ceará, Brasil \\ E-mail: marilia.furtado@aluno.uece.br \\ Ingrid Mikaela Moreira de Oliveira \\ ORCID: https://orcid.org/0000-0002-8901-362X \\ Universidade Estadual do Ceará, Brasil \\ E-mail: ingrid.moreira@aluno.uece.br \\ Vera Lúcia Mendes de Paula Pessoa \\ ORCID: https://orcid.org/0000-0002-5441-5311 \\ Universidade Estadual do Ceará, Brasil \\ E-mail: vera.mendes@uece.br \\ Ana Ruth Macedo Monteiro \\ ORCID: https://orcid.org/0000-0002-1130-1293 \\ Universidade Estadual do Ceará, Brasil \\ E-mail: anaruth.macedo@uece.br
}

\begin{abstract}
Resumo
Este estudo objetivou identificar, por meio da literatura científica, os cuidados multiprofissionais que são oferecidos aos pacientes em cuidados paliativos internados em unidades de terapia intensiva. Realizou-se uma revisão integrativa da literatura, com a inclusão de artigos originais que respondessem à questão de pesquisa, sem limitação de ano e ou de idioma. Como resultado, obteve-se um total de nove artigos incluídos para a síntese de estudos, sendo em sua maioria pesquisas de origem brasileira e de natureza qualitativa. Os artigos analisados possibilitaram a visão do cuidado a partir de diversas categorias profissionais, sendo este focado em ações de conforto fisico, como o alívio da dor e outros sintomas, seguido do suporte à esfera emocional. Apareceram, em menor frequência nos estudos, ações de apoio aos familiares, e o suporte religioso também esteve presente. Percebe-se que a multidisciplinaridade necessita ser estimulada em tais ambientes, com vistas à proporcionar um cuidado mais holístico.
\end{abstract}

Palavras-chave: Cuidados paliativos; Unidade de terapia intensiva; Equipe de assistência ao paciente.

\begin{abstract}
This study aimed to identify, in the scientific literature, the multidisciplinary care that is offered to patients in palliative care hospitalized in intensive care units. An integrative literature review was carried out, with the inclusion of original articles that answered the research question, without limitation of year and language. As a result, a total of nine articles were included for the synthesis of the studies, being mostly researches of Brazilian origin and of a qualitative nature. The articles analyzed enabled the vision of care from different professional categories, which focused on physical comfort actions, such as pain relief and other symptoms, followed by support for the emotional sphere. Actions to support families appeared less frequently in the studies, and religious support was present. It was possible to realize that the multidisciplinarity needs to be encouraged in such environments, providing, in such way, a more holistic care to the patients and family.
\end{abstract}

Keywords: Palliative care; Intensive care unit; Patient care team.

\section{Resumen}

Este estudio tuvo como objetivo identificar, en la literatura científica, los cuidados multidisciplinarios que se ofrecen a los pacientes en cuidados paliativos hospitalizados en unidades de cuidados intensivos. Se realizó una revisión integradora de la literatura, con la inclusión de artículos originales que respondieran a la pregunta de investigación, sin limitación de año e idioma. Como resultado, se incluyeron un total de nueve artículos para la síntesis de estudios, siendo en su mayoría investigaciones de origen brasileño y de carácter cualitativo. Los artículos analizados posibilitaron la visión del cuidado desde diferentes categorías profesionales, que se enfocaron en acciones de confort físico, como el alivio del dolor y otros síntomas, seguidas del apoyo a la esfera emocional. Las acciones de apoyo a 
las familias aparecieron con menos frecuencia en los estudios y hubo apoyo religioso. Se percibe que es necesario fomentar la multidisciplinariedad en dichos entornos, con miras a brindar una atención más integral.

Palabras clave: Cuidados paliativos; Unidade de cuidados intensivo; Grupo de atención al paciente.

\section{Introdução}

O fornecimento de cuidados a pacientes e familiares com necessidades paliativas demanda uma avaliação multidimensional dos aspectos físicos, sociais e espirituais, bem como dos valores e preferências individuais de cada paciente e sua rede de apoio. Para isso, a equipe multidisciplinar se torna essencial, através do trabalho conjunto com vistas a fornecer o melhor cuidado e de qualidade ao paciente (WHO, 2016). Tal equipe pode ser composta por profissionais de diversas áreas, como médicos, enfermeiros, fisioterapeutas, terapeutas ocupacionais, nutricionistas, psicólogos, farmacêuticos e assistentes sociais, ocorrendo inclusive a inclusão de assistentes religiosos. Tal diversidade de saberes objetiva oferecer um cuidado que se direcione para o indivíduo, e não apenas para o adoecimento, considerando cada paciente como um ser único e com um contexto social e familiar (Velasco \& Ribeiro, 2021).

Os cuidados paliativos podem ser fornecidos em qualquer nível de assistência, inclusive na Unidade de Terapia Intensiva (UTI). Apesar dos avanços científicos e tecnológicos, a UTI continua sendo um local com elevado número de óbitos (Pessini, 2016). Com a instalação da pandemia do novo coronavírus, denominado SARS-CoV-2, o número de óbitos em UTI teve aumento importante, tornando ainda mais relevante a atuação dos cuidados paliativos no âmbito de tais unidades, desde o momento do diagnóstico de uma doença grave e ameaçadora à vida (WHO, 2016).

Com vistas a realizar uma aproximação com o objeto de estudo, e revisar a literatura científica acerca do assunto, objetivou-se identificar os cuidados multiprofissionais que são oferecidos aos pacientes em cuidados paliativos internados em unidades de terapia intensiva.

\section{Metodologia}

Desenvolveu-se uma revisão integrativa da literatura, elaborada de acordo com as recomendações de Mendes, Silveira e Galvão (2008). Para construção da pergunta da pesquisa, utilizou-se o acrônimo População/problema, Fenômeno de Interesse e Contexto (PICo), recomendado pelo Instituto Joanna Briggs (Aromatis \& Munn, 2021). Assim, "P”= população adulta ou pediátrica em cuidados paliativos, "I" = Cuidados Multiprofissionais e "Co" = Unidade de Terapia Intensiva. Obteve-se, dessa maneira, a seguinte questão de pesquisa: Quais são os cuidados multiprofissionais oferecidos a pacientes em cuidados paliativos internados em Unidades de Terapia Intensiva?

Como critério de inclusão, optou-se por incluir artigos originais que respondessem à questão de pesquisa, sem limitação de ano ou idioma. Como critério de exclusão, os estudos indisponíveis para leitura na íntegra e de forma gratuita foram retirados.

A busca pelos estudos ocorreu de forma pareada, nas seguintes fontes de dados: Literatura Latino-Americana e do Caribe em Ciências da Saúde (LILACS), Science Direct, Web of Science, Scopus, National Library of Medicine via PubMed (MEDLINE/PubMed) e Scientific Electronic Library Online (SciELO). Os descritores foram utilizados de acordo com os termos indexados no Descritores em Ciências da Saúde (DeCS) e Medical Subject Headings (MeSH). Para tal, fez-se uso dos operadores booleanos "AND", "OR" e "NOT".

A estratégia de busca utilizada variou conforme a necessidade em cada base de dados, e está demonstrada no quadro a seguir. Ressalta-se que a etapa de seleção dos artigos nas bases de dados foi realizada com o auxílio do gerenciador de referências EndNote ${ }^{\circledR}$ Web, versão online e gratuita, para uma melhor uniformização na seleção dos dados, e maior potencial de reprodutibilidade do estudo, conforme recomendações (Mendes, Silveira \& Galvão, 2019). 
Quadro 1. Estratégia de busca dos artigos utilizada conforme as fontes de dados. Fortaleza, 2021.

\begin{tabular}{|c|c|}
\hline LILACS & 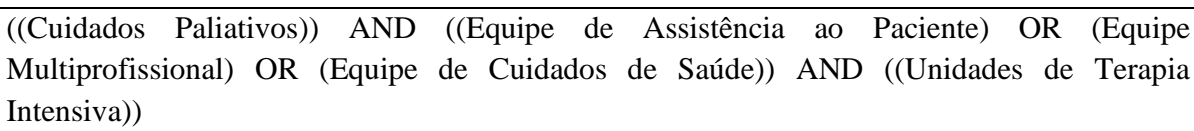 \\
\hline Science Direct & $\begin{array}{l}\text { (Patient Care Team) OR (Interdisciplinary Health Team) OR (Health Care Teams)) AND } \\
\text { (TITLE (Palliative Care) AND (Intensive Care Units)) }\end{array}$ \\
\hline Web of Science & $\begin{array}{l}\text { TI }=(\text { Palliative Care }) \text { AND ALL=(Patient Care Team OR Interdisciplinary Health Team OR } \\
\text { Health Care Teams) AND TI=(Intensive Care Units) }\end{array}$ \\
\hline Scopus & $\begin{array}{l}\text { ( TITLE ( ( "PALLIATIVECARE")) } \\
\text { "INTERDISCIPLINARY } \\
\text { HEALTH }\end{array}$ \\
\hline Scielo & $\begin{array}{l}\text { ((Cuidados Paliativos)) AND } \text { ((Equipe de Assistência ao Paciente) OR } \text { (Equipe } \\
\text { Multiprofissional) OR (Equipe de Cuidados de Saúde)) AND ((Unidades de Terapia } \\
\text { Intensiva)) }\end{array}$ \\
\hline MEDLINE/PubMED & $\begin{array}{l}((((\text { Palliative Care })[\text { Title] }) \text { AND }((\text { Patient Care Team) OR (Interdisciplinary Health Team) OR } \\
(\text { Health Care Teams }))) \text { AND ((Intensive Care Units)[Title]) }) \text { NOT ((Review)[Title] })\end{array}$ \\
\hline
\end{tabular}

Fonte: Autores.

O processo de busca e seleção dos artigos ocorreu durante os meses de maio e junho de 2021.

Para a extração dos dados dos estudos, utilizou-se do programa Microsoft Excel®, sendo documentados os dados relativos à identificação dos artigos (autores, país de origem, idioma, periódico, ano de publicação, objetivos, população, tipo de estudo, os cuidados relatados, e os profissionais envolvidos) bem como o nível de evidência. Os resultados foram compilados com a intenção de apresentar a visão global de todo o assunto e discutidos à luz da literatura pertinente. Por se tratar de estudo de revisão, não se fez necessário a aprovação do mesmo por comitê de ética em pesquisa.

\section{Resultados}

A busca nas fontes de dados resultou numa amostra inicial de 410 artigos, dos quais 79 foram removidos por estarem duplicados. Dos 331 estudos restantes, 297 foram retirados através da leitura dos títulos e resumos, por não se enquadrarem nos critérios de inclusão do estudo. Por fim, 34 artigos foram selecionados para análise através da leitura completa, sendo que desses, três estavam indisponíveis para leitura e 22 não responderam à pergunta de pesquisa, resultando assim num total de nove estudos considerados elegíveis para a síntese integrativa, conforme demonstrado na Figura 1. 
Figura 1. Fluxograma de busca e seleção dos artigos para inclusão na revisão integrativa. Fortaleza, 2021.

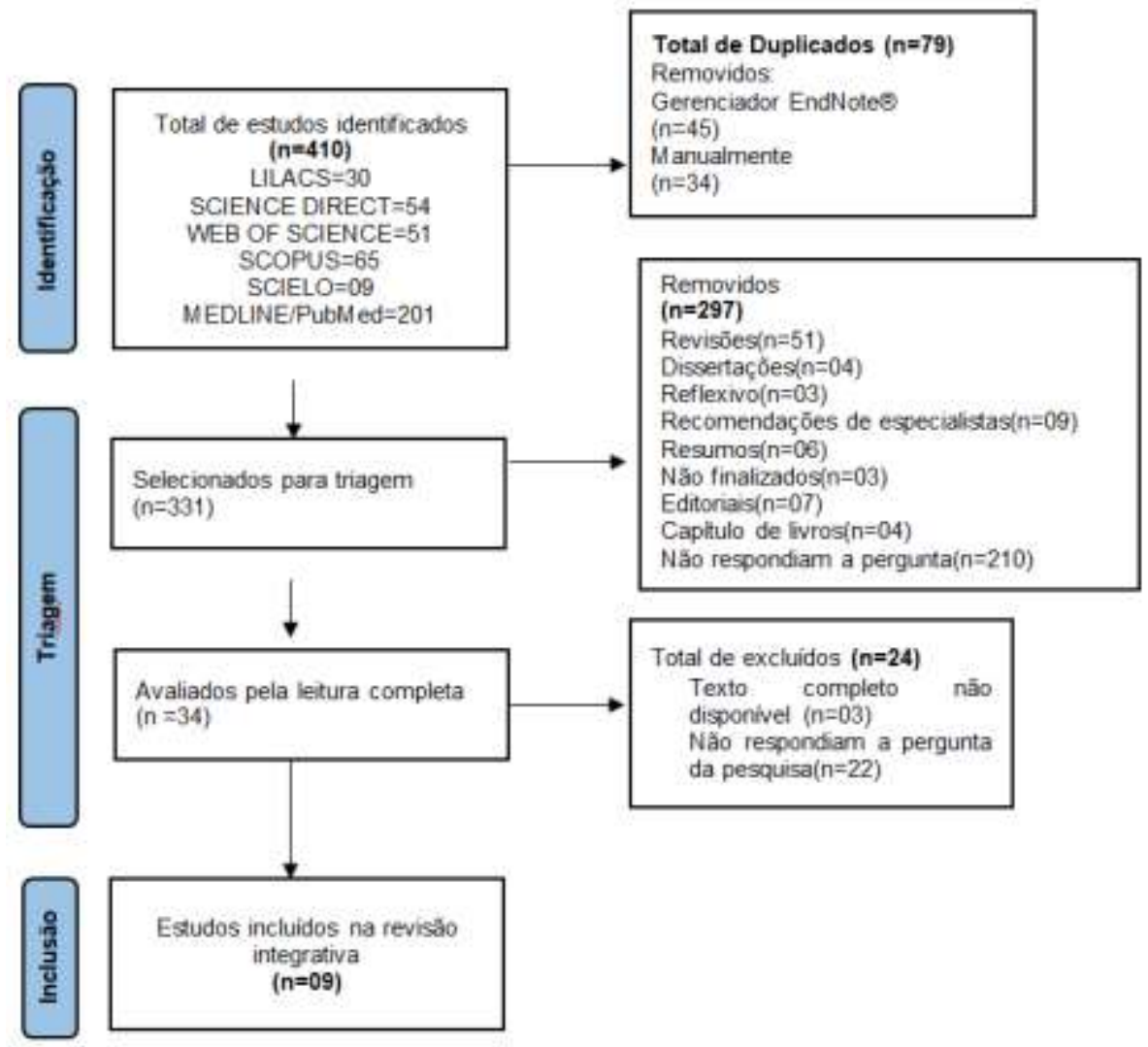

Fonte: Autores.

Os dados de caracterização dos artigos, como ano, país e tipo de estudo estão demonstrados no Quadro 2. Conforme visualizado, a maioria dos artigos é de origem nacional, publicado em periódicos brasileiros (Barros et al., 2013; Silva, Souza, Pedreira, Santos \& Faustino, 2013; Silva, Pereira \& Mussi, 2015; Souza, Miranda, Cruz \& Forte, 2016; Gulini et al., 2017; Pegoraro \& Paganini, 2019; Pires, Menezes \& Cerqueira, 2020) e disponíveis na língua nativa (Barros et al., 2013; Silva, Souza, Pedreira, Santos \& Faustino, 2013; Silva, Pereira \& Mussi, 2015; Gulini et al., 2017; Pegoraro \& Paganini, 2019; Pires, Menezes \& Cerqueira, 2020;). Houve um predomínio nas publicações referentes aos anos de 2013 (Silva, Souza, Pedreira, Santos \& Faustino, 2013; Barros et al., 2013), 2016 (Souza, Miranda, Cruz \& Forte, 2016; Wysham, Hochman, Wolf, Cox \& Kamal, 2016) e 2020 (Bobillo-Perez et al., 2020; Pires, Menezes \& Cerqueira, 2020), com dois artigos em cada período. Quanto à metodologia, percebe-se a predominância dos estudos de natureza qualitativa (Barros et al, 2013; Silva, Souza, Pedreira, Santos \& Faustino, 2013; Silva, Pereira \& Mussi, 2015; Gulini et al., 2017; Pegoraro \& Paganini, 2019; Pires, Menezes \& Cerqueira, 2020), exploratórios e descritivos (Barros et al., 2013; Silva, Souza, Pedreira, Santos \& Faustino, 2013; Pires, Menezes \& Cerqueira, 2020), com apenas três documentos de abordagem quantitativa (Souza, Miranda, Cruz \& Forte, 2016; Wysham, Hochman, Wolf, Cox \& Kamal, 2016; Bobillo-Perez et al., 2020). 
Quadro 2. Caracterização dos estudos incluídos na Revisão Integrativa. Fortaleza, 2021.

\begin{tabular}{|c|c|c|c|c|c|c|c|c|}
\hline Código & Autor & Ano & País & Idioma & Periódico & Tipo de Estudo & Metodologia & $\begin{array}{l}\text { Nível de } \\
\text { Evidência }\end{array}$ \\
\hline A1 & Silva et al. & 2013 & Brasil & Português & $\begin{array}{l}\text { Ciência e } \\
\text { Saúde Coletiva }\end{array}$ & $\begin{array}{l}\text { Exploratório e } \\
\text { descritivo }\end{array}$ & Qualitativa & 4 \\
\hline A2 & $\begin{array}{l}\text { Pegoraro } \\
\text { MM e } \\
\text { Paganini } \\
\text { MC }\end{array}$ & 2019 & Brasil & Português & $\begin{array}{l}\text { Revista } \\
\text { Bioética }\end{array}$ & $\begin{array}{l}\text { Não } \\
\text { especificado } \\
\text { pelos autores }\end{array}$ & Qualitativa & 4 \\
\hline A3 & Gulini et al. & 2017 & Brasil & Português & $\begin{array}{l}\text { Revista da } \\
\text { Escola de } \\
\text { Enfermagem } \\
\text { da USP }\end{array}$ & $\begin{array}{l}\text { Pesquisa } \\
\text { Convergente } \\
\text { Assistencial }\end{array}$ & Qualitativa & 4 \\
\hline A4 & Pires et al. & 2020 & Brasil & Português & $\begin{array}{l}\text { Acta Paulista } \\
\text { de } \\
\text { Enfermagem }\end{array}$ & $\begin{array}{l}\text { Exploratório e } \\
\text { descritivo }\end{array}$ & Qualitativo & 4 \\
\hline A5 & $\begin{array}{l}\text { Silva RS, } \\
\text { Pereira A e } \\
\text { Mussi FC. }\end{array}$ & 2015 & Brasil & Português & $\begin{array}{l}\text { Escola Anna } \\
\text { Nery Revista } \\
\text { de } \\
\text { Enfermagem }\end{array}$ & $\begin{array}{l}\text { Interacionismo } \\
\text { Simbólico }\end{array}$ & Qualitativo & 4 \\
\hline A6 & Barros et al. & 2013 & Brasil & Português & $\begin{array}{l}\text { Rev. } \\
\text { (Univ. } \\
\text { Estado Red. } \\
\text { Online J., }\end{array}$ & $\begin{array}{l}\text { Exploratório e } \\
\text { descritivo }\end{array}$ & Qualitativo & 4 \\
\hline A7 & $\begin{array}{l}\text { Bobillo- } \\
\text { Perez et al. }\end{array}$ & 2020 & $\begin{array}{l}\text { Não } \\
\text { identificado }\end{array}$ & Inglês & $\begin{array}{l}\text { BMC } \\
\text { Palliative Care }\end{array}$ & Caso-controle & Quantitativo & $3 a$ \\
\hline A8 & Souza et al. & 2016 & Brasil & Inglês & $\begin{array}{l}\text { Revista } \\
\text { Brasileira de } \\
\text { Terapia } \\
\text { Intensiva }\end{array}$ & $\begin{array}{l}\text { Coorte } \\
\text { retrospectivo }\end{array}$ & Quantitativo & $2 b$ \\
\hline A9 & $\begin{array}{l}\text { Wysham et } \\
\text { al. }\end{array}$ & 2016 & $\begin{array}{l}\text { Estados } \\
\text { Unidos }\end{array}$ & Inglês & $\begin{array}{l}\text { Journal of Pain } \\
\text { and Symptom } \\
\text { Management }\end{array}$ & Transversal & Quantitativo & 4 \\
\hline
\end{tabular}

Fonte: Autores.

Pela classificação de nível de evidências do modelo oficialmente aceito, proposto pelo Oxford Centre for EvidenceBased Medicine (2009), a maioria se enquadra como de nível 4, ou seja, evidência de estudos descritvos (não experimentais) ou com abordagem qualitativa. Ressalta-se que os estudos quantitativos incluídos não foram de intervenção, ou seja, não experimentais, pois o objetivo era avaliar como o cuidado é oferecido em sua forma mais natural possível.

Para melhor visualização dos objetivos e dos resultados dos estudos, estes foram organizados por meio do quadro 3, exposto a seguir. Observa-se que os objetivos de muitos dos estudos não eram de avaliar o cuidado em si, mas o conhecimento da equipe acerca dos CP, no entanto, nas falas dos participantes, era possível extrair dados relativos aos cuidados, pois estes chegavam à definição do objeto por meio de suas partes, tal como suas ações. 
Quadro 3. Objetivos dos estudos e cuidados paliativos multiprofissionais na visão dos profissionais incluídos nesta Revisão Integrativa. Fortaleza, 2021.

\begin{tabular}{|c|c|c|c|c|}
\hline Código & Autor & Objetivo & Cuidados relatados & $\begin{array}{l}\text { Profissionais } \\
\text { estudo }\end{array}$ \\
\hline A1 & Silva et al & 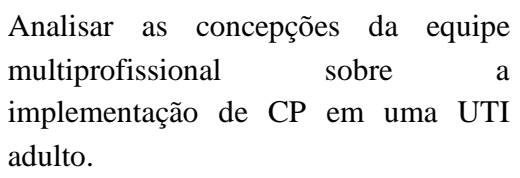 & $\begin{array}{l}\text { Foco em ações de cuidados físicos. A família } \\
\text { foi citada por um dos entrevistados. } \\
\text { Observou-se que tais pacientes são atendidos } \\
\text { por último. }\end{array}$ & $\begin{array}{l}\text { Enfermeiros, } \\
\text { fisioterapeutas, } \\
\text { médicos e } \\
\text { nutricionista. }\end{array}$ \\
\hline A2 & $\begin{array}{l}\text { Pegoraro } \\
\text { MM e } \\
\text { Paganini } \\
\text { MC }\end{array}$ & $\begin{array}{l}\text { Investigar o conhecimento da equipe } \\
\text { sobre os CP na limitação de suporte de } \\
\text { vida e construir ações } \\
\text { multidisciplinares para o cuidado ao } \\
\text { paciente }\end{array}$ & $\begin{array}{l}\text { Ações de conforto físico apareceram em } \\
\text { todas as categorias. O suporte emocional, } \\
\text { familiar, espiritual e religioso foi citado, } \\
\text { variando em cada categoria analisada. }\end{array}$ & $\begin{array}{l}\text { Técnicos de } \\
\text { enfermagem, } \\
\text { enfermeiros, médicos, } \\
\text { fisioterapeutas, } \\
\text { psicólogos, assistente } \\
\text { social e nutricionistas }\end{array}$ \\
\hline A3 & Gulini et al & $\begin{array}{l}\text { Conhecer a percepção dos profissionais } \\
\text { de saúde de uma UTI acerca do CP }\end{array}$ & $\begin{array}{l}\text { Ações em sua maioria de conforto físico. Foi } \\
\text { possível visualizar também ações de conforto } \\
\text { emocional. A família foi citada no aspecto de } \\
\text { preparação emocional. }\end{array}$ & $\begin{array}{l}\text { Médicos, enfermeiros, } \\
\text { técnicos de } \\
\text { enfermagem } \\
\text { fisioterapeutas. }\end{array}$ \\
\hline A4 & Pires et al & $\begin{array}{l}\text { Analisar a percepção da equipe } \\
\text { multiprofissional sobre conforto no } \\
\text { final de vida na terapia intensiva. }\end{array}$ & $\begin{array}{l}\text { O alívio da dor foi considerada central. } \\
\text { Ações para a promoção da paz e } \\
\text { tranquilidade também foram citadas. O } \\
\text { conforto psicológico foi relatado. A } \\
\text { aproximação da família e a assistência } \\
\text { religiosa apareceu no discurso dos } \\
\text { entrevistados. }\end{array}$ & $\begin{array}{l}\text { Médicos, enfermeiras, } \\
\text { fisioterapeutas, técnicos } \\
\text { de enfermagem, } \\
\text { nutricionistas, } \\
\text { assistentes sociais e } \\
\text { psicólogo }\end{array}$ \\
\hline A5 & $\begin{array}{l}\text { Silva RS, } \\
\text { Pereira A e } \\
\text { Mussi FC }\end{array}$ & $\begin{array}{l}\text { Conhecer o significado do cuidar de } \\
\text { enfermagem para uma boa morte. }\end{array}$ & $\begin{array}{l}\text { Foco maior no cuidado físico. O suporte } \\
\text { emocional ao paciente e família também foi } \\
\text { citado. }\end{array}$ & $\begin{array}{l}\text { Enfermeiros e técnicos } \\
\text { de enfermagem }\end{array}$ \\
\hline A6 & $\begin{array}{l}\text { Barros et } \\
\text { al }\end{array}$ & $\begin{array}{l}\text { Verificar a compreensão de enfermeiros } \\
\text { que atuam na UTI sobre os CP. }\end{array}$ & $\begin{array}{l}\text { Ações que proporcionam o conforto físico. A } \\
\text { interação com a família foi citada. }\end{array}$ & eiros \\
\hline A7 & $\begin{array}{l}\text { Bobillo- } \\
\text { Perez et al }\end{array}$ & $\begin{array}{l}\text { Descrever como o cuidado paliativo é } \\
\text { conduzido em uma UTI pediátrica e } \\
\text { como a implementação de uma equipe } \\
\text { paliativista mudou tais práticas no } \\
\text { setor. }\end{array}$ & $\begin{array}{l}\text { Ações de limitação de suporte de vida. A } \\
\text { retirada de suporte foi a mais frequente. Os } \\
\text { autores relataram o cuidado com sedação e } \\
\text { analgesia em termos de prescrição médica. }\end{array}$ & Médicos \\
\hline A8 & Souza et al & $\begin{array}{l}\text { Descrever as características dos } \\
\text { pacientes com HIV/AIDS admitidos em } \\
\text { uma UTI que foram avaliados pela } \\
\text { equipe de CP e comparar os cuidados } \\
\text { antes e após decidida a limitação de } \\
\text { suporte. }\end{array}$ & $\begin{array}{l}\text { Redução no uso de medicações e transfusões. } \\
\text { Não foram encontradas diferenças no uso de } \\
\text { drogas para sedação e analgesia. Ações de } \\
\text { retirada de suporte foram as mais frequentes. } \\
\text { Houveram reuniões familiares para } 46 \% \text { dos } \\
\text { pacientes. }\end{array}$ & Médicos \\
\hline A9 & $\begin{array}{l}\text { Wysham } \\
\text { et al }\end{array}$ & $\begin{array}{l}\text { Examinar a aderência a medidas de } \\
\text { qualidade de cuidados paliativos em } \\
\text { diversas UTIs dos Estados Unidos, } \\
\text { segundo critérios de uma escala } \\
\text { proposta, }\end{array}$ & $\begin{array}{l}\text { Foco do cuidado em ações de conforto físico. } \\
\text { O suporte espiritual também foi relevante. } \\
\text { Diretivas antecipadas de vontade apareceu } \\
\text { em } 35.8 \% \text { dos casos, e o suporte emocional } \\
\text { foi documentado. }\end{array}$ & Médicos \\
\hline
\end{tabular}


Conforme demonstrado, percebe-se que os estudos analisados possibilitaram a visão dos cuidados a partir das diversas categorias profissionais de saúde que atuam na assistência aos pacientes nas Unidades de Terapia Intensiva. Com maior frequência, foram incluídos nos estudos os enfermeiros e técnicos de enfermagem, seguida da classe médica.

A partir dos resultados dos artigos analisados, pôde-se perceber o oferecimento do cuidado multiprofissional ao paciente em CP a partir de duas principais categorias, as quais serão melhor discutidas a seguir.

\section{Discussão}

\section{Cuidado multiprofissional ao paciente em paliação no alívio do desconforto físico e emocional}

Os cuidados multiprofissionais oferecidos aos pacientes em cuidados paliativos abordado nos estudos, através das falas dos entrevistados ou da análise de prontuários, foram focados em ações, em sua maioria, de conforto físico (Barros et al., 2013; Silva, Souza, Pedreira, Santos \& Faustino, 2013; Silva, Pereira \& Mussi, 2015; Bobillo-Perez et al., 2016; Souza, Miranda, Cruz \& Forte, 2016; Wysham, Hochman, Wolf, Cox \& Kamal,2016; Gulini et al., 2017;; Pegoraro \& Paganini, 2019; Pires, Menezes \& Cerqueira, 2020), como: analgesia, sedação, tratamento de feridas, cuidados estéticos e higiênicos, oxigenioterapia, nutrição, dentre outros citados pelos autores. Ressalta-se que estes cuidados foram centrais para todas as categorias profissionais dos estudos, com exceção da psicologia e serviço social (Barros et al., 2013; Silva, Souza, Pedreira, Santos \& Faustino, 2013; Silva, Pereira \& Mussi, 2015; Gulini et al, 2017; Pegoraro \& Paganini, 2019; Pires, Menezes \& Cerqueira, 2020).

A dor é um dos sintomas mais frequentes em adultos e crianças com necessidades paliativas, com níveis moderados a severos. Juntamente com outros sintomas de natureza física, como perda de apetite, dificuldade para respirar, constipação ou diarréia, náuseas e outros, tais sintomatologias demandam uma atenção especial. No entanto, as medidas farmacológicas devem ser aliadas a tratamentos de ordem não-farmacológica, citados somente em dois estudos incluídos (Silva, Pereira \& Mussi, 2015; Gulini et al., 2017), objetivando um cuidado holístico (WHO, 2016).

Além disso, no cuidado da dor e em outros aspectos físicos têm de se levar em conta o conceito de "dor total", elaborado primeiramente por Cicely Saunders, na Inglaterra. Tal concepção entende que o processo doloroso carrega consigo uma cadeia de sentimentos no paciente em fim de vida, os quais advém da dor física, psíquica, social e espiritual. Outras dimensões foram sendo acrescentadas às iniciais propostas por Cicely, como a dor financeira, dor interpessoal e a dor familiar (Carvalho, 2009).

Como parte integrante do tratamento da dor total, faz-se necessário o suporte emocional, dizendo palavras reconfortantes, conversas, possibilitar ambiente acolhedor, diminuição de medos e ansiedade, dentre outras, os quais estiveram presentes nos artigos analisados (Silva, Pereira \& Mussi, 2015; Gulini et al., 2017; Pegoraro \& Paganini, 2019; Pires, Menezes \& Cerqueira, 2020).

O aspecto do cuidado emocional envolve a atenção a sintomas como ansiedades (Pires, Menezes \& Cerqueira, 2020), depressões e o delírio, e deve ser valorizado tanto quanto as ações de suporte ao aspecto físico (WHO, 2016). O cuidado com a confusão mental do paciente esteve presente, sendo citada pelos profissionais da área médica (Pegoraro \& Paganini, 2019). Além disso, o oferecimento de suporte para o enfrentamento da situação vivida foi também visualizado, como uma ação dos profissionais da psicologia (Pegoraro \& Paganini, 2019).

Uma das ferramentas muito utilizadas para o fornecimento do suporte emocional diz respeito à escuta ativa, bem como outras estratégias verbais e não verbais de comunicação (Araújo \& Silva, 2012), como o oferecimento de carinho (Gulini et al., 2017; Pegoraro \& Paganini, 2019; Pires, Menezes \& Cerqueira, 2020) uma conversa (Gulini et al, 2017). A escuta ativa, no contexto dos CP configura-se como uma estratégia fundamental para que o cuidado seja centrado no paciente, procurando silenciar as diversas vozes internas do sujeito que cuida, permitindo uma abertura ao outro, em suas reais necessidades 
(Higuera, 2005). Desta forma, o uso de ferramentas verbais e não-verbais de comunicação interpessoal, como o toque (Pires, Menezes \& Cerqueira, 2020), permitem a transmissão de acolhimento e compaixão, sendo de grande relevância para o cuidado ao paciente em fim de vida, e deve fazer parte do arcabouço profissional de saúde que lide com tais indivíduos (Araújo \& Silva, 2012).

Para uma assistência integral ao paciente em CP deve-se sempre levar em consideração que o indíviduo é um ser multidimensional, tanto do ponto de vista biológico, como psicológico, social e espiritual. Para que o cuidado ao paciente nessa fase de vida seja holístico, o relacionamento interpessoal, com empatia e transmissão de afetividade, é um subsídio de valor considerável (Araújo \& Silva, 2007). Estratégias como a realização de atividades lúdicas, como citadas no estudo A5 (Silva, Pereira \& Mussi, 2015) trazem o humor como uma opção espontânea para o alívio de tensões em momentos de sofrimento (Araújo \& Silva, 2007).

O cuidado à esfera emocional do paciente em terminalidade mostra-se como parte indispensável a uma assistência de saúde baseada nos princípios da integralidade e da humanização, bem como demonstra a importância a todas as esferas de dimensões do indivíduo assistido, possibilitando ações em saúde holísticas.

\section{Abordagem familiar do indivíduo e a questão da fé}

O cuidado com a família, por meio do acolhimento e promoção da tranquilidade, conversas e orientações, alívio de angústias, visita em horários flexíveis, conforto emocional e condução de reuniões famíliares também esteve presente nos artigos analisados (Barros et al., 2013; Silva, Pereira \& Mussi, 2015; Souza, Miranda, Cruz \& Forte 2016; Gulini et al., 2017; Pegoraro \& Paganini, 2019).

O suporte aos familiares dos pacientes que enfrentam o processo de morte é um aspecto fundamental dos $\mathrm{CP}$, com uma avaliação cuidadosa das necessidades de tais cuidadores e o pleno desenvolvimento de um plano de assistência diferenciado. A família normalmente apresenta problemas específicos, bem como necessidades não atendidas, estando propensa ao desenvolvimento de morbidades psicológicas e físicas, como depressão e ansiedade. Tais cuidadores são frequentemente sobrecarregados, inclusive nos aspectos financeiros, tornando-se muitas vezes isolados socialmente. Em muitos casos, a família possui necessidades de cuidado ainda maiores do que as do próprio paciente em si (WHO, 2016). Ressalta-se que apenas um dos estudos analisados trouxe a visão da necessidade de apoio à família devido ao seu abalo emocional (Silva, Pereira \& Mussi, 2015). Em relação aos psicológos, estes demonstraram ações para o auxílio dos familiares no enfrentamento da situação e do processo de luto antecipado, e o serviço social ressaltou o apoio quanto aos trâmites legais após o óbito (Pegoraro \& Paganini, 2019), trazendo assim o reconhecimento da necessidade de suporte em alguns aspectos específicos da sobrecarga familiar.

A necessidade de cada família designa qual a melhor estratégia de cuidado a ser oferecida. As famílias valorizam como suporte o próprio fato de saber que as necessidades do seu ente querido estão sendo atendidas, bem como ao serem asseguradas de que os profissionais de saúde são empáticos e respeitosos com seu parente (O’Sullivan Alvariza, Ohlén, \& Larsdotter, 2021). Nesse sentido, a enfermagem demonstrou a preocupação em atender tais demandas, integrando a família ao cuidado, tornando visíveis as intervenções realizadas, com o objetivo do promover tranquilidade (Silva, Pereira \& Mussi, 2015; Pegoraro \& Paganini, 2019;). Além disso, receber informações claras e precisas e o auxílio para reconhecer a aproximação da morte são também fatores considerados positivos, ações essas demonstradas em algum dos estudos incluídos (Pegoraro \& Paganini, 2019; Gulini et al., 2017). O acompanhamento após o óbito, através de ligações telefônicas, ou acompanhamentos presenciais são extremamente valorizados pelos indivíduos, principalmente quando oferecido por profissionais que participaram ativamente do processo de morte do paciente (O’Sullivan Alvariza,, Ohlén, \& Larsdotter, 2021). 
Ressalta-se que nesse aspecto de suporte após o óbito, nenhum dos artigos incluídos citou tal ação, com exceção do suporte aos trâmites legais, oferecido pelo serviço social (Pegoraro \& Paganini, 2019).

Por fim, a assistência religiosa esteve presente, embora esta tenha sido citada somente em três dos artigos analisados, como no auxílio para a manutenção das crenças pessoais, o apoio religioso e possibilitar a presença do sacerdote na UTI (Wysham, Hochman, Wolf, Cox \& Kamal, 2016; Pegoraro \& Paganini, 2019; Pires, Menezes \& Cerqueira, 2020).

A religiosidade encontra-se em um entrelaçamento com a espiritualidade, embora não sejam sinônimos, visto que a religião representa a adesão a concepções e crenças, vinculadas em geral a uma instituição ou doutrina (Barbosa \& Freitas, 2009). No contexto do sofrimento das doenças ameaçadoras à vida, a religiosidade auxilia no enfrentamento da situação, preparando os indivíduos para as adversidades, como um recurso para lidar com a doença e com a morte. Neste cenário, o profissional de saúde possui o papel de manter-se sensível, respeitando a fé do paciente (Gulini et al., 2017) e permitir a livre expressão da religiosidade, como possibilitar a entrada de objetos religiosos no leito do paciente na UTI (Pires, Menezes \& Cerqueira, 2020), bem como buscando favorecer a leveza na expressão de crenças e diminuir os sentimentos de culpa, porém permitindo o encontro do indivíduo com sua espiritualidade e religião (Barbosa \& Freitas, 2009).

A espiritualidade diz respeito a uma ampla dimensão da vida humana, que traduz a forma como os sujeitos agem em relação às experiências de vida, se expressam ou buscam significados, seus objetivos e transcedências, bem como estes se concectam aos momentos, a si mesmos, aos outros e ao sagrado (Nolan, Saltmarsh \& Leget, 2011). A espiritualidade esteve presente nos estudos incluídos, através de conversas que objetivavam o alcance da paz, proporcionando um ambiente acolhedor (Pegoraro \& Paganini, 2019; Pires, Menezes \& Cerqueira, 2020) e possibilitando momentos de encontro com a natureza (Pires, Menezes \& Cerqueira, 2020).

Segundo um estudo de revisão sistemática realizado em 2019, pacientes, familiares e profissionais frequentemente entendem a espiritualidade como a ocorrência de relacionamentos significativos, sendo necessário o entendimento do conceito para sua correta aplicação no cenário dos CP. Os participantes dos estudos visualizavam que tal cuidado estava sendo ofertado por meio da presença oferecida, na atenção à espiritualidade e esperança de seus entes queridos, e ter suas famílias e pacientes sendo encorajados e elevados a momentos de paz (Gijsberts, Liefbroer, Otten \& Olsman, 2019).

Percebe-se, dessa maneira, que o apoio à expressão religiosa e proporcionar momentos de paz e tranquilidade aos pacientes e familiares deve fazer parte do escopo de ação dos profissionais que lidam com indíviduos com necessidades paliativas, possibilitando o encontro com a espiritualidade e a ressignificação de sofrimentos.

\section{Conclusão}

A partir da leitura dos artigos incluídos nesta revisão integrativa, pôde-se perceber que os cuidados multiprofissionais aos pacientes em cuidados paliativos de unidades de terapia intensiva ainda estão focados em ações de cuidado à esfera física.

Contudo, percebe-se que o conforto emocional aos pacientes e familiares vem se tornando mais frequente, abrangendo atitudes como o toque, o oferecimento de palavras de apoio e a realização de atividades lúdicas. Tal resultado vai de encontro com o preconizado com a literatura paliativista, devido à necessidade real de tais indivíduos que enfrentam doenças ameaçadoras e o sofrimento emocional advindo destas situações.

Além disso, a assistência à espiritualidade e à religiosidade de tais pacientes e familiares possibilita o encontro dos indivíduos com sua fé, a ressignificação de acontecimentos, bem como a aceitação da doença e o encontro de sentidos.

A ampliação do escopo de ações da equipe ao paciente crítico em cuidados paliativos é possibilitada pela presença da multidisciplinaridade, que deve ser estimulada e implementada em tais setores, com vistas a um cuidado holístico e direcionado às diversas necessidades dos indivíduos que enfrentam doenças graves. 
Sugere-se a realização de pesquisas que avaliem as principais barreiras para a implementação do cuidado paliativo integral em unidades de terapia intensiva pela equipe multiprofissional, possibilitando que intervenções possam ser realizadas e que o escopo de ação em tais unidades seja ampliado.

\section{Referências}

Araújo, M. M. T. \& Silva, M. J. P. (2007) A comunicação com o paciente em cuidados paliativos: valorizando a alegria e o otimismo. Rev Esc Enferm USP, 41(4):668-74.

https://www.scielo.br/j/reeusp/a/pCsdGFyV45fnyQmNpTGh5Bz/?lang=pt\&format=pdf\#: :text=A\%20conversa\%20amig\%C3\%A1vel\%20e\%20emp\%C3\%A 1 tica,fora $\% 20 \mathrm{de} \% 20$ possibilidades\%20de\%20cura.

Araújo, M. M. T. \& Silva, M. J. P. (2012) O conhecimento de estratégias de comunicação no atendimento à dimensão emocional em cuidados paliativos. Texto Contexto Enferm., 21(1): 121-9. https://www.scielo.br/j/tce/a/vpS9FyhFCgFLbtGjnVBQVLK/?format=pdf\&lang=pt.

Araújo, M. M. T. \& Silva, M. J. P. (2012) Estratégias de comunicação utilizadas por profissionais de saúde na atenção à pacientes sob cuidados paliativos. Rev Esc Enferm USP, 46(3):626-32. https://www.scielo.br/j/reeusp/a/PkND3TttB3sCS8d9jWQkLGQ/?lang=pt\&format=pdf.

Barbosa, K. A. \& Freitas, M. H. (2009) Religiosidade e atitude diante da morte em idosos sob cuidados paliativos. Rev. Kairós., 12 (1): $113-134$. https://revistas.pucsp.br/index.php/kairos/article/view/2783/1818.

Barros, N. C., Alves, E. R., Oliveira, C. D., Dias, M. D., França, I. S. X. \& Freire, M. E. M. (2013) Cuidados Paliativos na UTI: compreensão dos enfermeiros. Rev. Pesqui. (Univ. Fed. Estado Rio J., Online), 5(1):3293-01. doi: https://doi.org/10.5902/217976925857.

Bobillo-Perez, S., Susana, S., Girona-Alarcon, M., Felipe, A., Balaguer, M., Hernandez-Platero L., Sole-Ribalta, A., Guitart, C., Jordan, I. \& Cambra, F. J. End-of-life care in a pediatric intensive care unit: the impact of the development of a palliative care unit. BMC Palliative Care, 2020; 19:74. https://bmcpalliatcare.biomedcentral.com/articles/10.1186/s12904-020-00575-4.

Carvalho, M. M. (2009) A dor do adoecer e do morrer. Acad. Paul. Psicol; vol.29 no.2 São Paulo. http://pepsic.bvsalud.org/scielo.php?script=sci_arttext\&pid=S1415-711X2009000200009\&lng=pt\&nrm=iso.

Centre for evidence-based medicine. (2009). Oxford Centre for Evidence-based Medicine: Levels of evidence (March 2009). https://www.cebm.ox.ac.uk/resources/levels-of-evidence/oxford-centre-for-evidence-based-medicine-levels-of-evidence-march-2009.

Gijsberts, m. j. h. e., Liefbroer, a. i., Otten, r., \& Olsman, e. (2019) Spiritual care in palliative care: a systematic review of the recent european literature. Med sci(basel),7(2):25. doi: 10.3390/medsci7020025.

Gulini, J. H. M. B; Nascimento, E. R. P; Moritz, R. D; Rosa, L. M., Silveira, N. R., \& Vargas, M. A. O. (2017) A equipe da Unidade de Terapia Intensiva frente ao cuidado paliativo: discurso do sujeito coletivo. Rev Esc Enferm USP, 51:e03221. doi: http://dx.doi.org/10.1590/S1980-220X2016041703221.

Higuera, J. C. B. (2005) La escucha ativa em cuidados paliativos. Rev Est Med Hum [on-line]. https://arsmedica.cl/index.php/MED/article/view/209/142.

Mendes, K. D., Silveira, R. C., \& Galvão, C. M. (2008) Revisão integrativa: método de pesquisa para a incorporação de evidências na saúde e na enfermagem. Texto \& contexto enferm, 17(4): 758-64. https://www.scielo.br/j/tce/a/XzFkq6tjWs4wHNqNjKJLkXQ/?lang=pt\&format=pdf

Mendes, K. D., Silveira, R. C. \& Galvão, C. M. (2019) Uso de gerenciador de referências bibliográficas na seleção dos estudos primários em revisão integrativa. Texto \& contexto enferm, v. 28: e20170204. doi: https://dx.doi.org/10.1590/1980-265X-TCE-2017-0204.

Nolan, S., Saltmarsh, P., \& Leget, C. (2011) Spiritual care in palliative care: Working towards an EAPC Task Force. Eur. J. Palliat. Care,18:86-89. https://www.eapcnet.eu/wp-content/uploads/2021/03/EJPC-2011-18-2.pdf.

O'sullivan, A., Alvariza, A., Ohlén, J., \& Larsdotter, C. (2021) Support received by family members before, at and after an ill person's death. BMC Palliat Care., 20, 92. doi: https://doi.org/10.1186/s12904-021-00800-8.

Pegoraro, M. M., \& Paganini M. C. (2019) Cuidados paliativos e limitação de suporte de vida em terapia intensiv. Rev. Bioét,; 27 (4): 699-710. doi: https://doi.org/10.1590/1983-80422019274353.

Pessini, L. (2016) Vida e morte na UTI: a ética no fio da navalha. Rev. bioét. (Impr.); 24 (1): 54-63. https://www.scielo.br/j/bioet/a/TZNdxQ5McVJDSTBr7yWvTMS/?format=pdf\&lang=pt.

Pires, I. B., Menezes, T. M. O., \& Cerqueira, B. B. (2020) Conforto no final de vida na terapia intensiva: percepção da equipe multiprofissional. Acta Paul Enferm, 33; 1-7. doi: http://dx.doi.org/10.37689/actaape/ 2020AO0148.

Silva, C. C., Souza, D.M.; Pedreira, LC, Santos, M. R., \& Faustino, T. N. (2013) Concepções da equipe multiprofissional sobre a implementação dos cuidados paliativos na unidade de terapia intensiva. Cien Saude Colet, 8(9):2597-2604. doi: https://doi.org/10.1590/S1413-81232013000900014

Silva, R. S., Pereira, A, \& Mussi, F. C. (2015) Conforto para uma boa morte: perspectiva de uma equipe de enfermagem intensivista. Esc Anna Nery,19(1):4046. doi: https://doi.org/10.5935/1414-8145.20150006.

Souza, P. N., Miranda, E.J. P, Cruz, R., \& Forte, D. N. (2016) Palliative care for patients with HIV/AIDS admitted to intensive care units. Rev Bras Ter Intensiva, 28(3):301-309. doi: 10.5935/0103-507X.20160054.

Velasco T. D., Ribeiro, S. C. (editores). (2021) Cuidados paliativos na emergência. 1 ed. Barueri. Manole. 
Research, Society and Development, v. 10, n. 15, e393101522852, 2021

(CC BY 4.0) | ISSN 2525-3409 | DOI: http://dx.doi.org/10.33448/rsd-v10i15.22852

World Health Organization. (2016) Planning and implementing palliative care services: a guide for programme managers. Geneva. https://apps.who.int/iris/handle/10665/250584.

Wysham, N. G., Hochman, M. J., Wolf, S. P., Cox, C. E. \& Kamal, A. H. (2016) Performance of Consultative Palliative Care Model in Achieving Quality Metrics in the ICU. J Pain Symptom Manage, 52(6):873-877. doi: 10.1016/j.jpainsymman.2016.05.026. 